\section{The Genetics of Cancer}

\section{GENES ASSOCIATED WITH CANCER INVASION, METASTASIS AND CELL PROLIFERATION}

\section{G.V. Sherbet \& M.S. Lakshmi Academic Press, \$85, 352 pp. ISBN: 0.126-39875-5, 1997}

ReVIEWEd By Daniel A. HabeR

Massachusetts General Hospital Cancer Center and Harvard Medical School, Charlestown, MA 02129

The Genetics of Cancer: Genes Associated With Cancer Invasion, Metastasis and Cell Proliferation, by G.V. Sherbet and M.S. Lakshmi, provides a broad review of many of the genetic pathways implicated in the development of cancer. The book discusses the clonal evolution of cancer cells, cellcycle regulation, apoptosis, angiogenesis, invasion and metastasis, and more. The book's greatest strength is in compiling and extensively referencing the massive and often paradoxical clinical literature correlating the expression patterns of cancer genes with different tumor phenotypes. However, although it offers a very comprehensive listing of much work published in the field, this book gives only limited critical appraisal, and even contains some incongruities and a number of striking inaccuracies.

For example, it lists contradictory observations on the ability of wild-type p53 to induce expression of the epidermal growth factor receptor (EGFr), while discussing the correlation in tumors between p53 mutations and EGFr overexpression. It also describes the role of the MCC gene in genetic predisposition to colon cancer, even though the correct gene was subsequently shown to be the neighboring APC gene. And there are significant omissions or inaccuracies in the description of important genes, such as BRCA1, p16 INK4a, APC and MDM2. Perhaps such mistakes reflect the difficult task of two authors reviewing a very broad field, as the best chapters are those dealing with the pathways involved in metastasis and invasion, a subject with which the authors are most familiar.

In addition, the chapters dealing with cell-cycle regulation and cancer predisposition are less than current-a risk in- herent in these rapidly advancing fields of research. Recent discoveries in DNA mismatch repair and angiogenesis also are not well covered. Nonetheless, by its very breadth, this book provides a compendium of clinical correlates in cancer genetics, and its emphasis on cancer progression and metastasis serves to highlight the new frontier in this field.

Until now, much of cancer genetics has focused on the early events in malignant transformation. Mutations in genes such as $\mathrm{RB}, \mathrm{APC}, \mathrm{WT} 1$ and BRCA1 may be present in the germline and serve as initiating events in tumorigenesis. In many cases, these genes were identified by positional cloning strategies, using familial cancer syndromes, and their contribution to the develop-

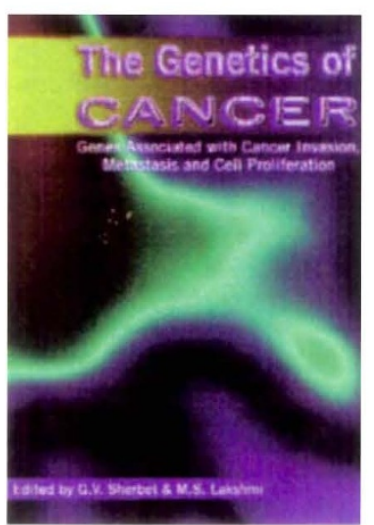

which to define the anticipated flood of candidate genes involved in cancer progression, invasiveness and metastasis? As noted by the authors, current functional assays using animal models have serious limitations. Much of the existing literature, as compiled in this book, consists of correlative analyses of expression differences between normal tissues and their derived cancers. Interpretation of such comparisons is difficult, as the cell of origin of a cancer is likely to be a rare stem cell, rather than the more common, terminally differentiated cell type present in the mature organ.

Differences in expression patterns between distinct cell types may provide only limited insight into the changes ment of cancer was confirmed by the presence of inactivating mutations.

Recent approaches, including comparative genomic hybridization, representational difference analysis, and the use of micro-arrayed probes for mRNA expression profiling ('DNA chips'), target the large number of differences between normal and cancer cells, most of which result from genetic events arising in the course of tumor progression. Furthermore, therapeutic interventions aimed at such secondary genetic targets, such as anti-angiogenic peptides or inhibitors of telomerase, may prove as efficacious as those directed at the initiating genetic lesions.

What then are the standards by that occur during malignant transformation. With the exception of rare chromosomal translocations, genes whose expression levels are altered in cancer cells have not been demonstrated to have genetic alterations in their promoters, pointing to potentially complex regulatory mechanisms. Definitive evidence that a newly discovered gene plays an important role in tumor progression therefore depends on the demonstration of mutations or other genetic alterations that lead to either activation or disruption of gene function. The authors conclude by asking whether any known gene can be definitively labeled as a 'metastasis gene'. Their answer is no. Much work remains to be done.

\section{ERRATUM}

Tissue transglutaminase selectively modifies gliadin peptides that are recognized by gut-derived T cells in celiac disease

Øyind Molberc, Stephen N. Mcadam, Roman Körner, Hanne Quarsten, Christel Kristiansen, lars Madsen, lars Fugcer, Helge Scott, Ove Norén, Peter Roepstorff, Knut E.A. Lundin, HANS SJÖSTRÖM \& LUDVIC M. SOLID

Nature Med. 4, 713-717, 1998

The corresponding author was incorrectly listed as K.E.A.L. The correct corresponding author is $\varnothing$.M.

On page 714 , right column, the third sentence of paragraph 3 should read "Although the binding affinity to DQ2 of acid/heat treated peptide 138-152; E148 was similar to peptide 138-152; E140, E148, E1 50 the latter was a more potent antigen for the T-cell transfectant (Fig. 4).

We regret these errors. 